\title{
ZOONOTIC StREPTOCOCCUS CANIS INFECTION IN SINGAPORE
}

Singapore Med J 2016; 57(4): 218 doi: 10.11622/smedj.2016077

Dear Sir,

Streptococcus dysgalactiae (S. dysgalactiae) subspecies equisimilis is a Lancefield Group G streptococcus (GGS) that is responsible for a variety of human infections, including pharyngitis, skin and soft-tissue infections, septic arthritis, osteomyelitis, and bacteraemia. Streptococcus canis (S. canis), a phenotypically similar close relative, causes pyogenic infections in dogs and cats. However, zoonotic $S$. canis infections in humans have been reported.(1) It has been suggested that the actual number of $S$. canis infections in humans may be underestimated, as GGS is not routinely identified at the species level. ${ }^{(1)}$

According to the Agri-Food and Veterinary Authority of Singapore, the number of dog licences issued in Singapore increased from 35,125 in 2000 to 60,440 in 2014. ${ }^{(2)}$ Our study aimed to investigate whether $S$. canis formed a significant proportion of GGS isolated in our laboratory. All large-colony beta-haemolytic GGS isolated for a period of one year, from 19 August 2013 to 19 August 2014, were identified at the species level using MALDI-TOF (Bruker Singapore Pte Ltd, Singapore). Any suspected S. canis isolates were confirmed by sequencing of the 165 rDNA gene.

During the study period, 181 GGS were isolated. The majority were identified as $S$. dysgalactiae subsp. equisimilis. One isolate (DM12402_14) was identified by MALDI-TOF as Streptococcus castoreus with a score of 2.273. The second- and third-choice identifications were $S$. canis and $S$. dysgalactiae subsp. dysgalactiae, with scores of 2.249 and 2.150, respectively. The isolate DM12402_14 was identified by API 20 Strep (bioMérieux Singapore Pte Ltd, Singapore) as most likely to be S. canis (99.1\%, Profile 0063405), with $S$. dysgalactiae subsp. equisimilis $(0.7 \%)$ as the second-choice identification. The VITEK® 2 (bioMérieux Singapore Pte Ltd, Singapore) identified the DM12402_14 isolate as S. dysgalactiae with 97\% probability. However, S. canis is not included in the VITEK 2 database. A $1.3 \mathrm{~kb}$ fragment of the $16 S$ rRNA gene was amplified, sequenced and submitted to the National Center for Biotechnology Information's Basic Local Alignment Search Tool and the 16SpathDB 2.0 database. The closest matches were with S. canis (99\% Genbank accession number EU075063.1, 99.8\% Genbank accession number DQ303184.1.1).

There were two limitations to this study. Firstly, only three $S$. canis reference spectra exist in the MALDI-TOF database (MBT 3.0). Secondly, $16 S$ rRNA gene sequencing was not performed on all GGS. Therefore, it is possible that some $S$. canis were missed.

DM12402_14 was isolated in a mixed culture with Neisseria canis from the hand of a 20-year-old man. The patient sustained multiple abrasions and puncture wounds over his right thumb and thenar eminence after his hand was caught in the wheel of a bicycle during an accident. He presented the following day with cellulitis of the right hand. His wounds were debrided and he completed a course of oral amoxicillin and clavulanate potassium with good outcome. Although the patient did not volunteer any information about animal contact at presentation, he recalled at follow-up, after the culture results were known, that his wounds had come into contact with his pet dog, a Siberian husky.

S. canis is not a common cause of GGS infection in humans in Singapore. Although misidentification at the species level does not greatly affect the treatment, availability of low-cost methods of bacterial identification like MALDI-TOF may reveal previously unsuspected zoonotic causes of streptococcal soft-tissue infections of the upper limb.

Yours sincerely,

Ruth En Si $\underline{\operatorname{Tan}}^{1,2}$, Wearn Xin $\underline{\mathrm{Yee}}^{3}$, Delphine Yan Hong $\underline{\mathrm{CaO}^{3}}$, Peck Lay $\underline{\mathrm{Tan}}^{3}$, Tse Hsien $\underline{\mathrm{Koh}}^{3}$

${ }^{1}$ Department of Hand Surgery, ${ }^{2}$ Department of Emergency Medicine, ${ }^{3}$ Department of Pathology, Singapore General Hospital, Singapore. koh.tse.hsien@singhealth.com.sg

\section{RefERENCES}

1. Galpérine T, Cazorla C, Blanchard E, Boineau F, Ragnaud JM, et al. Streptococcus canis infections in humans: retrospective study of 54 patients. J Infect 2007; 55:23-6.

2. Agri-Food \& Veterinary Authority of Singapore. Annual Report 2014/15. Available at: http://www.ava.gov.sg/docs/default-source/publication/annual-report/ ava-2014-2015-ar. Accessed March 15, 2016 